\title{
Brillouin Fiber Laser Utilizing Nonlinear Birefringent Photonic Crystal Fiber (PCF)
}

\author{
Meri Algarni \\ Department of Physics \\ School of Arts and Science \\ Clark Atlanta University, Atlanta, GA, USA
}

\begin{abstract}
A Brillouin fiber laser utilizing an exceedingly nonlinear birefringent photonic precious stone fiber is accounted for. The center of the fiber was curved fit as a fiddle. A solitary longitudinal-mode high power fiber laser was utilized as a Brillouin pump, and the laser created multi-wave length yield with a $0.04 \mathrm{~nm}$ partition. Modifying the polarization controller plates made it conceivable to get a single wavelength laser. The impact of the little center birefringent photonic gem fiber on the yield of the laser was investigated. The laser was steady and demonstrated a power change of under $0.2 \mathrm{~dB}$. The yield of the laser was checked utilizing an optical range analyzer of determination $0.01 \mathrm{~nm}$.
\end{abstract}

\section{General Terms}

Fiber Laser

\section{Keywords}

Fiber Laser, Brillouin Fiber laser, photonic precious stone fiber, nonlinear optics, single-mode laser

\section{INTRODUCTION}

Empowered Brillouin dispersing happened when the optical control propelled into the fiber surpasses a Brillouin edge level. The SBS detrimentally affects gadgets given very nonlinear fiber due to the immersion impact of information power [1]. Be that as it may, for various applications, for example, enhancement, lasing, moderate light era, and detecting, SBS is exceptionally helpful [2-7]. By and large, a more extended length of single-mode fiber (SMF) is required to create gadgets utilizing the SBS impact, because of its low nonlinear coefficient. As of late, strands with high nonlinear coefficients have been utilized to create photonic gadgets with an incredibly diminished length [2-8]. Nonlinear PCF is extremely alluring for creating photonic gadgets for their exceptional optical properties. Late examinations concerning SBS in exceptionally birefringent photonic precious stone fiber have found that the fiber is a decent possibility for creating fiber lasers and sensors. Multiwavelength fiber laser sources are extremely alluring for their applications in optical interchanges, photonic gadget portrayals, and detecting. A few procedures have been proposed and exhibited to deliver a multiwavelength fiber laser utilizing an erbium-doped fiber as an increased medium; SBS is one of these procedures. In this letter, a double wavelength Brillouin fiber laser in a unidirectional ring cavity setup utilizing a very nonlinear, polarization-keeping up photonic gem fiber is illustrated. An erbium/ytterbium co-doped high power fiber laser, composed and developed in-house, was utilized as a pump. Assist, the impacts of the polarization-keeping up the property of the
PCF and modular (longitudinal) structure of the pump laser on the yield of the Brillouin laser are tentatively researched.

\section{LITERATURE REVIEW}

\subsection{Photonics Crystal Fibers}

Photonic-Crystal Fiber (PCF) is another class of optical fiber in light of the properties of photonic gems. Given its capacity to limit light in empty centers or with containment qualities unrealistic in customary optical fiber, PCF is currently discovering applications in fiber-optic interchanges, fiber lasers, nonlinear gadgets, high-control transmission, profoundly touchy gas sensors, and different ranges. More particular classes of PCF incorporate photonic-bandgap fiber (PCFs that bind light by band crevice impacts), holey fiber (PCFs utilizing air openings in their cross-areas), gap helped fiber (PCFs directing light by a traditional higher-record center changed by the nearness of air gaps), and Bragg fiber (photonic-bandgap fiber shaped by concentric rings of multilayer film). Photonic precious stone strands might be viewed as a subgroup of a more broad class of microstructure optical filaments, where light is guided by basic changes, and not just by refractive file contrasts.

\subsection{Optical Fibers}

Optical Fibers have developed into many structures since the down to earth achievements that saw their more extensive presentation in the 1970s as customary stride list fibers [1][2] and later as single material filaments where engendering was characterized by a powerful air cladding structure.[3] When all is said in done, customary organized strands, for example, photonic precious stone filaments, have a cross-segment (typically uniform along the fiber length) microstructured from one, two or more materials, most normally masterminded occasionally over a great part of the cross-area, more often than not as a "cladding" encompassing a center (or a few centers) where light is limited. For instance, the filaments initially exhibited by Russell comprised of a hexagonal cross section of air gaps in a silica fiber, with a strong (1996) or empty (1998) center at the inside where light is guided. Different courses of action incorporate concentric rings of at least two materials, initially proposed as "Bragg filaments" by Yeh and Yariv (1978), a variation of which was as of late manufactured by Temelkuran et al. (2002) and others. The most reduced announced weakening of strong center photonic precious stone fiber is $0.37 \mathrm{~dB} / \mathrm{km},[4]$ and for the empty center is $1.2 \mathrm{~dB} / \mathrm{km}[5]$. An optical fiber or optical fiber is an adaptable, straightforward fiber made by drawing glass (silica) or plastic to a measurement somewhat thicker than that of a human hair.[1] Optical strands are frequently utilized as a way to transmit light between the two finishes of the fiber and find wide use in fiber-optic interchanges, where 
they allow transmission over longer separations and at higher transfer speeds (information rates) than wire links. Filaments are utilized rather than metal wires since signs go along them with lesser measures of misfortune; what's more, strands are likewise safe to electromagnetic impedance, an issue from which metal wires endure excessively.[2] Fibers are additionally utilized for light and are wrapped in groups so they might be utilized to convey pictures, in this way permitting seeing in restricted spaces, as on account of a fiberscope.[3] Specially outlined strands are additionally utilized for an assortment of different applications, some of them being fiber optic sensors and fiber lasers.

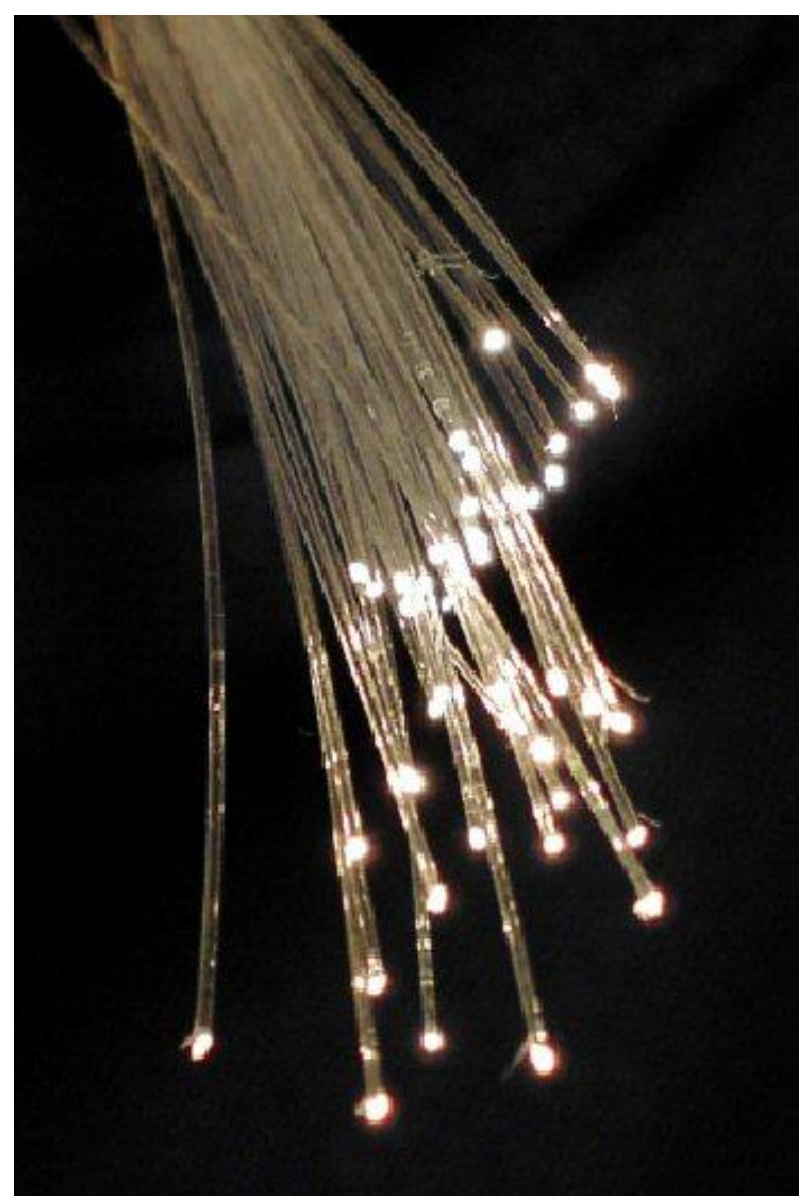

Figure1: A Bundle of Optical Fibers

\subsection{Methods of operation}

Photonic Crystal Fibers can be partitioned into two methods of operation, as indicated by their instrument for control. Those with a strong center, or a center with a higher normal file than the microstructured cladding, can work on a similar file controlling rule as routine optical fiber - anyway, they can have a significantly higher powerful refractive record differentiate amongst center and cladding, and subsequently can have considerably more grounded restriction for applications in nonlinear optical gadgets, polarization-looking after filaments, (or they can likewise be made with much lower successful list differentiate). Then again, one can make a "photonic bandgap" fiber, in which the light is kept by a photonic bandgap made by the microstructured cladding such a bandgap, legitimately composed, can restrict light in a lower-list center and even an empty (air) center. Bandgap filaments with empty centers can conceivably go around cutoff points forced by accessible materials, for instance, to make strands that guide light in wavelengths for which straightforward materials are not accessible (because the light is essentially noticeable all around, not in the strong materials). Another potentially preferred standpoint of an empty center is that one can powerfully bring materials into the center, for example, a gas that will be broke down for the nearness of some substance. PCF can likewise be changed by covering the gaps with sol-gels of comparative or distinctive file material to upgrade its transmittance of light.
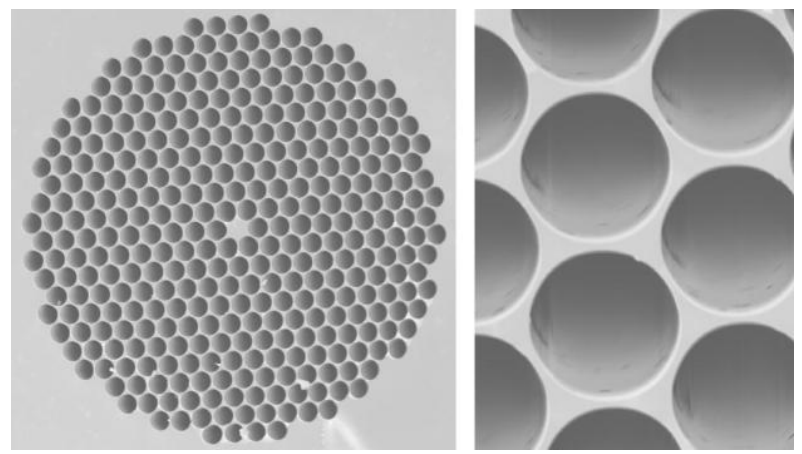

Figure2: SEM micrographs of a photonic-crystal fiber created at US Naval Research Laboratory. (left) The measurement of the strong center at the focal point of the fiber is $5 \mu \mathrm{m}$, while (right) the width of the gaps is $4 \mu \mathrm{m}$.

\subsection{Optical Fibers Cable Types}

The following are the most famous Optical Fibers cables

Table1: Most Famous Optical Fibers Cables

\begin{tabular}{|l|l|}
\hline & Type \\
\hline OFC & Optical Fiber Conductive \\
\hline OFN & Optical Fiber Nonconductive \\
\hline OFCG & $\begin{array}{l}\text { Optical Fiber Conductive } \\
\text { General use. }\end{array}$ \\
\hline OFNG & $\begin{array}{l}\text { Optical Fiber Nonconductive } \\
\text { General use }\end{array}$ \\
\hline OFCP & $\begin{array}{l}\text { Optical Fiber Conductive, } \\
\text { Plenum }\end{array}$ \\
\hline OFNP & $\begin{array}{l}\text { Optical Fiber Nonconductive } \\
\text { Plenum }\end{array}$ \\
\hline OFCR & $\begin{array}{l}\text { Optical Fiber Conductive, } \\
\text { Riser }\end{array}$ \\
\hline OFNR & $\begin{array}{l}\text { Optical Fiber Nonconductive } \\
\text { Riser }\end{array}$ \\
\hline OPGW & $\begin{array}{l}\text { Optical Fiber Composite } \\
\text { Overhead Ground Wire }\end{array}$ \\
\hline
\end{tabular}

\section{RESULTS AND TECHNIQUES}

The schematic of the laser appears in Figure 1. The ring resonator comprises a three-port polarization-autonomous circulator, a three-meter-long nonlinear circular center photonic gem fiber, a polarization controller to control the polarization condition of the light waves inside the hole, and a 3 - $\mathrm{dB}$ coupler to extricate the yield of the laser. The PCF has a 
nonlinear coefficient of $0.12 \mathrm{~m}-1 \mathrm{~W}-1$, center measurement of $1.3 \times 2.3 \mu \mathrm{m}$, the birefringence of $2.2 \times 10-3$ at $1530 \mathrm{~nm}$, constriction of $84 \mathrm{~dB} / \mathrm{km}$ at $1550 \mathrm{~nm}$ (which gives a viable length of $2.91 \mathrm{~m}$ ), and a beat length of $0.7 \mathrm{~mm}$. The Brillouin pump laser (input laser) could create a solitary longitudinalmode laser with a yield force of $450 \mathrm{~mW}$. Figure 2 demonstrates the modular structure of the pump laser got utilizing a checking FabryPerot range analyzer (SFPSA, $\mathrm{FSR}=2 \mathrm{GHz}$ ) of determination $6.7 \mathrm{MHz}$, which affirmed the single-longitudinal-mode wavering of the laser. The pump laser was infused into the Brillouin laser pit in the clockwise heading. The Brillouin wavelengths were created in the anticlockwise heading and were removed through a $3-\mathrm{dB}$ coupler. The yield of the laser was checked utilizing an optical range analyzer of determination $0.01 \mathrm{~nm}$. The yield control from the forward spreading waves inside the hole was checked utilizing the control meter[9].

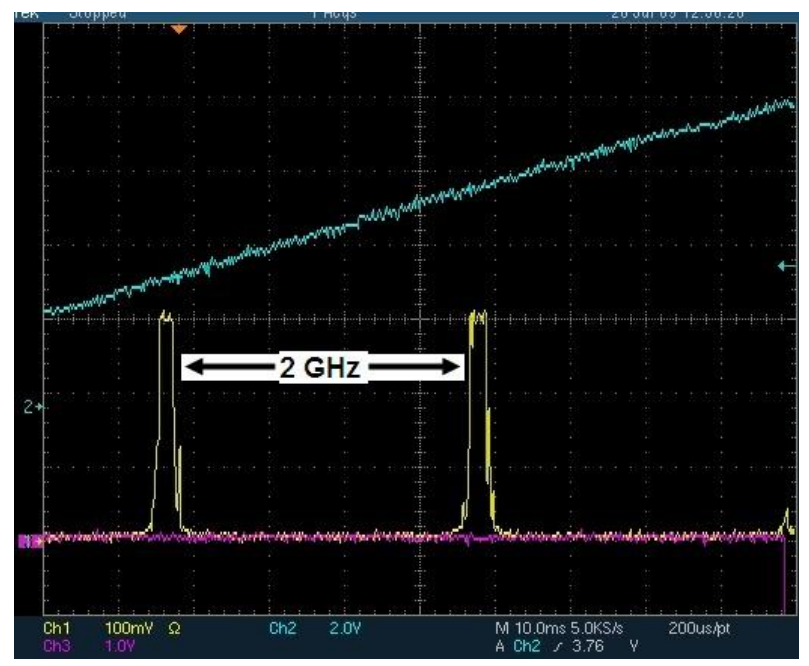

Figure 3: Modal structure, utilizing an SFPSA and oscilloscope, of the information pump laser at $1565.96 \mathrm{~nm}$ with yield force of $\sim 450 \mathrm{Mw}$. Adapted by Jonas $\mathrm{K}$. Valiunas and Gautam Das

\section{CONCLUSION}

All in all, the creators have shown a Brillouin fiber laser utilizing exceptionally nonlinear PCF. The laser could create a multiwavelength yield with a division of $0.04 \mathrm{~nm}$. The different pinnacles were expected to the multimode property of the little center PCF utilized as a part of this test. Through change of the polarization controller plates, the laser could be made to waver in single-wavelength or double wavelength administrations, where the division between two lasing wavelengths depends on the birefringence property of the
PCF. The high nonlinearity of the PCF decreased the length of the full pit, which brought about more extensive longitudinal mode dividing. The birefringent property of the PCF expanded the dependability of the laser.

\section{ACKNOWLEDGMENTS}

The author would like to thank Saudi Arabian Culutral Mission (SACM) for the finincially support through Al-Baha University.

\section{REFERENCES}

[1] S. Bickham, A. Kobyakov, and S. Li, "Nonlinear Optical Fibers with Increased SBS Thresholds," OFC, 2006.

[2] K. S. Abedin, "Brillouin amplification and lasing in a single-mode $\mathrm{AS}(2) \mathrm{Se}(3)$ chalcogenide fiber," Optics Letters, vol. 31, no. 11, pp. 1615-1617, June 2006.

[3] G. S. Qin, A. Mori, and Y. Ohishi, "Brillouin lasing in a single-mode tellurite fiber," Optics Letters, vol. 32, no. 15, pp. 2179-2181, Aug.2007.

[4] P. Bayvel and I. P. Giles, "Evaluation of Performance Parameters of Single-Mode All-Fiber Brillouin Ring Lasers," Optics Letters, vol. 14, no. 11, pp. 581-583, June 1989.

[5] A. Yeniay, J. M. Delavaux, and J. Toulouse, "Spontaneous and stimulated Brillouin scattering gain spectra in optical fibers," Journal of Lightwave Technology, vol. 20, no. 8, pp. 1425-1432, Aug.2002.

[6] K. Y. Song, W. W. Zou, Z. Y. He, and K. Hotate, "Alloptical dynamic grating generation based on Brillouin scattering in polarization-maintaining fiber," Optics Letters, vol. 33, no. 9, pp. 926-928, May 2008.

[7] T. R. Parker, M. Farhadiroushan, V. A. Handerek, and A. J. Rogers, "Temperature and strain dependence of the power level and frequency of spontaneous Brillouin scattering in optical fibers," Optics Letters, vol. 22, no. 11, pp. 787-789, June 1997.

[8] G. S. Qin, H. Sotobayashi, M. Tsuchiya, A. Mori, T. Suzuki, and Y. Ohishi, "Stimulated Brillouin scattering in a single-mode tellurite fiber for amplification, lasing, and slow light generation," Journal of Lightwave Technology, vol. 26, no. 5-8, pp. 492-498, Mar.2008.

[9] Jonas K Valiunas and Gautam Das. Article: Brillouin Fiber Laser using Nonlinear Birefringent Photonic Crystal Fiber. IJCA Proceedings on International Conference on Emerging Trends in Informatics and Communication ICETIC 2016(1):43-45, September 2016. 